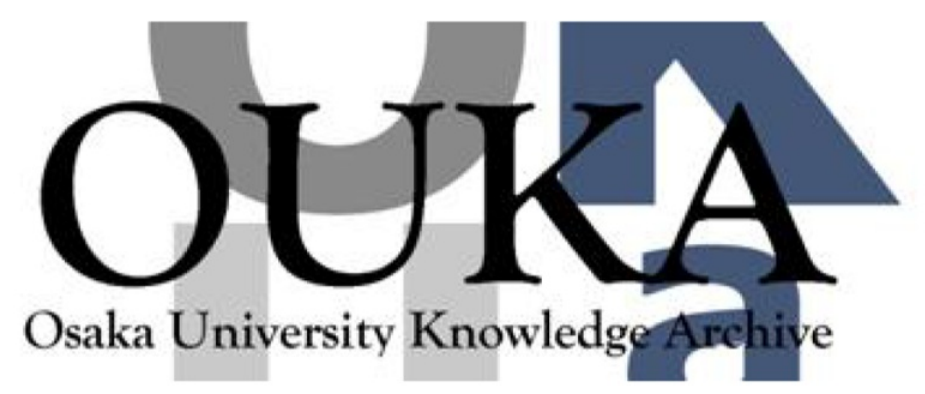

\begin{tabular}{|c|l|}
\hline Title & $\begin{array}{l}\text { Single-Particle Light Scattering Study of } \\
\text { Polyethyleneglycol-Grafted Poly(ureaurethane) } \\
\text { Microcapsule in Ethanol }\end{array}$ \\
\hline Author(s) & $\begin{array}{l}\text { Ohsawa, Akane; Mori, Yasutaka; Narita, Takayuki } \\
\text { et al. }\end{array}$ \\
\hline Citation & $\begin{array}{l}\text { Colloids and Surfaces B: Biointerfaces. 37(3-4) } \\
\text { p. 129-p. 132 }\end{array}$ \\
\hline Issue Date & 2004-09-01 \\
\hline oaire:version & AM \\
\hline URL & https://hdl. handle.net/11094/25937 \\
\hline rights & \\
\hline Note & \\
\hline
\end{tabular}

Osaka University Knowledge Archive : OUKA

https://ir. Library. osaka-u. ac. jp/

Osaka University 


\title{
Single Particle Light Scattering Study of Polyethyleneglycol-Grafted Poly(ureaurethane) Microcapsule in Ethanol
}

Ken Terao, ${ }^{\mathrm{a}, *}$ Akane Ohsawa, ${ }^{\mathrm{a}}$ Yasutaka Mori, ${ }^{\mathrm{a}}$ Takayuki Narita, ${ }^{\mathrm{a}}$ Kimio Ichikawa, ${ }^{\mathrm{b}}$ and Toshiaki Dobashi ${ }^{\mathrm{a}}$

${ }^{a}$ Department of Biological and Chemical Engineering, Faculty of Engineering, Gunma University, 1-5-1, Tenjin-cho, Kiryu, Gunma 376-8515, Japan.

${ }^{\mathrm{b}}$ Fujinomiya Laboratory, Fuji Photo Film Company Ltd., 200 Ohnakazato, Fujinomiya, Shizuoka 418-8666, Japan.

*Corresponding author. Phone:+81-277-30-1478, Fax: +81-277-30-1401

E-mail: terao@bce.gunma-u.ac.jp (K. Terao)

\begin{abstract}
Microcapsules having polyethyleneglycol-grafted poly(ureaurethane) (PUU) membrane and di-2ethylhexyl phthalate core have been prepared, and the structure when they were suspended in dispersing ethanol have been studied by means of single-particle light scattering method. The PUU membrane was synthesized from monomers with aromatic functional groups (microcapsule MC110) and hexamethylene functional groups (microcapsule MC160). Because the outer and inner solvent passed through the membrane easily, the outside and inside the membrane were the same at the equilibrium state. The thickness of the wall membrane was significantly smaller than that calculated from the overall weight ratio of the wall-forming material and the core solvents. It was attributed to low affinity of PUU membranes and ethanol.
\end{abstract}

Key Words: microcapsule, size distribution, light scattering, permeability, dispersible 


\section{Introduction}

Poly(ureaurethane) (PUU) microcapsules are one of the most useful microcapsules manufactured from o/w emulsions by interfacial polymerization [1]. The suspension of the microcapsules in aqueous solutions are easily stabilized by a protective colloids such as copoly(vinylalchohol-vinylacetate). When the microcapsules are in contact with organic solvents, the inner oil core is gradually oozed and was replaced by the outer solvents. [2] It is expected that the microcapsules could be used as a minute reactor (microreactor) when the polymer chains confined in the microcapsule core were reacted with a reagent penetrating there because only low molecules pass through the wall membrane. Indeed, the diffusion coefficient of dye molecules depended strongly on the molecular size of the dye. [2(d)] If the microcapsules could be dispersed in organic solvents, applications as a microreactor would be extended to a variety of fields. However, it is difficult to disperse the microcapsules in non-aqueous solutions and protective colloids result in complicated effects in organic solvents, frequently. [3] Recently, Wakata and Ichikawa devised a PUU microcapsules with polyethylene glycol (PEG) chains on the surface. [4] We found that this microcapsule could be dispersed not only in water but also in a kind of organic solvents. It is interesting to study the structure of the microcapsules and the permeability characteristics of the microcapsule membrane in organic solvents as a first step to apply PUU microcapsules as microreactors shown in Fig. 1. The single particle light scattering technique is appropriate for determining the structure of the microcapsule, because this technique simplifies the complications that comes from a polydisperse system. [5] In this study, we prepared two kinds of PUU microcapsules dispersed in ethanol, determined both the thickness and the outer diameter of the microcapsules and evaluated the permeability characteristics of the wall membranes. 


\section{Materials and Methods}

\section{Preparation of a Microcapsules}

An adduct of trimethylolpropane carbamate with xylylene diisocyanate (TCXDI) and polyethylene glycol monomethyl ether (PEGME) was obtained as follows. $35 \mathrm{~g}$ of PEGME (average molecular weight: 5,000, Polyscience) dried in vacuum over night were dissolved into $35 \mathrm{~cm}^{3}$ of dried acetonitrile (Kanto) adding $4 \mathrm{~g}$ of molecular sieves $4 \mathrm{~A}$. After the mixture was stirred for 3 hours under dry nitrogen, $40 \mathrm{~g}$ of Takenate D110N (70 80\% ethyl acetate solution of TCXDI, Mitsui Takeda Chemical Co.) and $0.16 \mathrm{~cm}^{3}$ of Tin(II) 2ethylhexanoate (Wako) was added to the mixture. The resultant mixture was stirred at room temperature for an hour and at $50^{\circ} \mathrm{C}$ for 3 hours in order to complete the reaction.

$1.0 \mathrm{~g}$ of the mixture obtained and $1.0 \mathrm{~g}$ of Takenate D110N were added to $3.0 \mathrm{~g}$ of ethyl acetate (Wako) with $1.0 \mathrm{~g}$ of di-2-ethylhexyl phthalate (DOP, Wako). After stirring, we added $10 \mathrm{~g}$ of pure water and immersed vigorously at 5,000 rpm for ten minutes by using an emulsifier (Excel Auto, Nihon Seiki Co.). The emulsion was stirred at $40^{\circ} \mathrm{C}$ for $4 \mathrm{~h}$. It was confirmed that the most part of acetonitrile and ethyl acetate were evaporated in this process. The microcapsules thus manufactured is, hereafter, designated as MC110. A part of the chemical structure of the microcapsules is illustrated in Fig. 2. In the present synthesis, the mol ratio of TCXDI to PEGME was about 25 and the volume ratio $\phi$ of DOP to the wallforming materials was roughly estimated to be 1.30 using the densities of DOP and PUU membrane being 0.981 and 1.30 , respectively. [6]

Another microcapsules were prepared in the same manner as described above except for the following conditions. Instead of Takenate D110N, Takenate D160N (70 80\% ethyl acetate solution of trimethylolpropane, carbamate with hexamethylene diisocyanate, TCHDI) gifted from Marubeni Chemix Co. was used. The adduct of Takenate D160N and PEGME was prepared from $20 \mathrm{~g}$ of Takenate D160N, $40 \mathrm{~cm}^{3}$ of acetonitrile, and $20 \mathrm{~g}$ of PEGME with 
stirred at $60^{\circ} \mathrm{C}$ for $22 \mathrm{~h}$. The emulsion obtained was stirred at $50^{\circ} \mathrm{C}$ for $12 \mathrm{~h}$ after the emulsification. However, the surface of the suspension was covered with a thin film. This phenomenon suggests that some colloids were broken during the stirring and the colloids formed the film. The microcapsule made from Takenate D160N is designated as MC160.

\section{Characterization Methods of microcapsules}

$20 \mu \mathrm{l}$ of the microcapsule suspensions was poured into $10 \mathrm{ml}$ in methanol $(\mathrm{MeOH})$, ethanol $(\mathrm{EtOH})$, acetonitrile (Ac), tetrahydrofuran (THF), or 2-propanol. The suspension was centrifuged to obtain the supernatant after soaking in $30 \mathrm{ml}$ of the same organic solvent. The absorbance of the supernatant solution at $274 \mathrm{~nm}$ wavelength did not depend on the soaking time after three days. Thus, we regarded the absorbance of the supernatant solution obtained after soaking for three days as the equilibrium value. The release ratio defined by the weight ratio of DOP in the microcapsule core before and after soaking in organic solvents was estimated from the absorbances. The size distribution for each microcapsule was estimated from micrographs and about $500 \sim 1,000$ droplets (about 10 pictures) were counted in each run.

Single-particle light scattering measurements were carried out at $632.8 \mathrm{~nm}$ wavelength for MC110 and MC160 in EtOH with a laboratory made light scattering apparatus [7] and the thickness and the outer diameter of an arbitrarily chosen microcapsule were estimated. Each relative scattering intensity $I$ was determined as a function of scattering angle $\theta$ or the magnitude of the scattering vector $q\left[=\left(4 \pi / \lambda^{\prime}\right) \sin ^{2}(\theta / 2)\right]$ with $\lambda^{\prime}$ being the wavelength of the light in the medium. For the spherical shells, the particle scattering function $P(q)$ which is proportional to $I(q)$ is expressed as [8] 


$$
\begin{aligned}
& P(q)=\frac{9 \pi}{2}\left\{\frac{J_{3 / 2}\left(r_{\mathrm{o}} q\right)}{\left(r_{\mathrm{o}} q\right)^{3 / 2}}-\frac{\left(1-\delta / r_{\mathrm{o}}\right)^{3} J_{3 / 2}\left[\left(r_{\mathrm{o}}-\delta\right) q\right]}{\left[\left(r_{\mathrm{o}}-\delta\right) q\right]^{3 / 2}}\right\}^{2} \\
& J_{3 / 2}(r q)=\sqrt{2 / \pi}(r q)^{-3 / 2}[\sin (r q)-r q \cos (r q)]
\end{aligned}
$$

Here, $r_{\mathrm{o}}$ and $\delta$ are the outer radius of the shell and the thickness of the wall membrane, respectively. $\quad r_{\mathrm{o}}$ and $\delta$ of an arbitrarily chosen microcapsule were estimated by fitting the observed scattering curves to Eqs. (1) and (2). Details of the apparatus and analytical method are described in ref 7.

\section{Results and Discussion}

The microcapsules were dispersed completely in ethanol, at least for several months, whereas they aggregated in 2-propanol and sank at the bottom. Table 1 shows the release ratios for various organic solvents. The release ratios for the microcapsules MC110 with the medium of $\mathrm{EtOH}, \mathrm{MeOH}$ and $\mathrm{Ac}$ are unity within the experimental error. Thus, DOP contained in the core was completely replaced by the dispersing media. On the other hand, the release ratio for MC110 with THF is 0.9 . This means that $10 \%$ of DOP remained in the core than in the outer dispersing medium (c $\sim 0)$. The difference of DOP concentration at the equilibrium state suggests that a certain amount of impurity such as oligomers of TCXDI remains in the core, since the concentration gradient through the membrane can not be achieved by the DOP-THF binary systems in both sides of the membrane. For MC160, each release ratio was about $20 \%$ smaller than that for MC110. One of the reasons should be the loss of the colloids during the synthesis described in the experimental section. Therefore, it is suggested for the same reason that for MC160 the core DOP is completely replaced by outer $\mathrm{EtOH}$ and $\mathrm{MeOH}$, but certain amount of impurity is dissolved in the core when THF or Ac is used as the outer dispersing medium. 
Fig. 3 shows the size distribution of the microcapsule MC110 in the varius solvents. It is seen that the size distribution of MC110 in THF and Ac were appreciably smaller than that in water, $\mathrm{MeOH}$, and $\mathrm{EtOH}$ and almost as large as unfilled circles for the microcapsule redispersed in pure water after soaking in $\mathrm{EtOH}$. On the other hand, the size distribution of MC160 is almost independent of dispersing organic solvent, as shown in Fig. 4. The result shows that the swelling or shrinking behavior of wall membrane strongly depend on the small difference of the chemical structure of the membrane. All the organic solvents used in this study are good solvents and poor solvents of PUU. Thus, the microcapsule membrane should be more or less shrunken by soaking them in the solvents.

Four examples of the relative scattering intensity $I(q)$ of the microcapsules MC110 and $\mathrm{MC160}$ in EtOH are illustrated in Fig. 5 and 6, respectively. Angular dependence of the intensity for each microcapsule has several peaks characteristics to sphere particles / spherical shells. Because DOP is replaced completely by EtOH, the microcapsule can be modeled by the spherical shells with the same dispersing medium both the inside and the outside the shell. We analyzed the observed data by a least-squares method fit to eqs (1) and (2). The estimated parameters $\left(r_{\mathrm{o}}\right.$ and $\delta$ ) for each microcapsule are indicated in each panel of Figs. 5 and 6. The theoretical curves calculated with the parameters reasonably agrees with the experimental ones. The estimated ratios of $\delta$ to $r_{\mathrm{o}}$ were $0.11 \pm 0.01$ and $0.10 \pm 0.01$ for MC110 and MC160, respectively.

If all the monomer molecules are contributed to the wall membrane polymer network and the polymer neither swells nor shrinks, the ratio of $\delta$ to $r_{\mathrm{o}}$ is given by

$$
\delta / r_{\mathrm{o}}=1-\sqrt[3]{\phi /(1+\phi)}
$$

The ratios $\delta / r_{\mathrm{o}}=0.10 \sim 0.11$ determined by the scattering method are significantly smaller 
than 0.17 calculated by this equation with the preparation condition of $\phi=1.3$. These results support the shrinking of PUU membrane in the dispersing solvents.

\section{Conclusions}

We have manufactured poly(ureaurethane) microcapsules having polyethylenegrycol chains on the surface. The microcapsules were well dispersed in various organic solvents. Their dimensional properties and permeability have been investigated in four organic solvents and the following conclusions have been derived. (1) DOP core replaced by EtOH and $\mathrm{MeOH}$ when the microcapsules were dispersed in the solvent. (2) The wall membrane of MC110 in methanol and ethanol do not shrink as highly as that in water, tetrahydrofuran, and acetonitrile whereas the diameter of MC160 was independent of the dispersing organic solvent. (3) The membrane thickness estimated from a single-particle light scattering method was significantly smaller than those calculated from the volume ratio of the core material to the wall-forming material, which can be attributed to poor affinity of PUU membrane and the organic solvents.

\section{Acknowledgement}

This research was partially supported by Japan Society for the Promotion of Science Grant-in-Aid for Exploratory Research, 13875119 and by Ministry of Education, Culture, Sports, Science, and Technology (MEXT) Grant-in-Aid for Young Scientists (B), 14750703.

[1] T. Kondo J. Oleo Sci., 50 (2001) 143.

[2] (a) T. Yamamoto, T. Dobashi, M. Kimura and C.-P. Chang, Colloids and Surfaces. B: Biointerfaces, 25 (2002) 305. (b) T. Sato, S. Shibako, T. Yamamoto, K. Ichikawa and T. Dobashi, J. Membr. Sci., 213 (2003) 25. (c) C.-P. Chang, T. Yamamoto, M. Kimura, T. Sato, K. Ichikawa and T. Dobashi, J. Controlled Release. 86 (2003) 207. (d) T. Furukawa, S. 
Hung, T. Yamamoto, K. Terao, K. Ichikawa, and T. Dobashi, T. Trans. Mater. Res. Soc. Jpn., $28(2003) 989$.

[3] C.-P. Chang, M. Kimura, T. Yamamoto, M. Nobe, M. and T. Dobashi, Colloids and Surfaces. B: Biointerfaces, 30 (2004) 123.

[4] (a) Y. Wakata and K. Ichikawa, (Fuji Film Co.). Jpn. Kokai Tokkyo Koho, 10 114,153, May 6, 1998. (b) U. S. Patent 5,916,680, June 29, 1999.

[5] T. Dobashi, B. Chu, in M. J. Milling (Ed.), Surface Characterization Method, Marcel Decker: New York, 1999, Chapter 8.

[6] (a) T. Dobashi, H. Ishimaru, A. Sakanishi and K. Ichikawa, Langmuir, 3071 (2003) 19.

(b) T. Dobashi, T. Furukawa, K. Ichikawa and T. Narita, Langmuir, 18 (2002) 6031.

[7] T. Dobashi, T. Narita, J. Masuda, K. Makino, T. Mogi, H. Ohshima, M. Takenaka and B. Chu, Langmuir, 14 (1998) 745.

[8] M. Kerker, J. P. Kratohvil and E. Matijevic, J. Opt. Sci. Am., 52 (1962) 551. 
Table 1. Release ratio of DOP from the microcapsule

\begin{tabular}{lcccc}
\hline & \multicolumn{4}{c}{ Dispersant } \\
\cline { 2 - 5 } & EtOH & MeOH & THF & Ac \\
\cline { 2 - 5 } Microcapsule & \multicolumn{4}{c}{ Release ratio ${ }^{\text {a }}$} \\
\hline MC110 & $1.0_{2}$ & $1.0_{6}$ & $0.8_{7}$ & $0.9_{8}$ \\
MC160 & $0.7_{1}$ & $0.8_{1}$ & $0.6_{7}$ & $0.6_{4}$ \\
\hline
\end{tabular}

${ }^{\mathrm{a}}$ Determined from absorbance of the supernatant at $274 \mathrm{~nm}$ wavelength 


\section{Figure Caption}

Fig. 1. Schematic representation of exchange of the inner medium.

Fig. 2. A part of chemical structure of PUU microcapsule.

Fig. 3. Size distribution of the microcapsules MC110 in water (filled circles), $\mathrm{MeOH}$ (filled squares), EtOH (filled triangles), THF (unfilled triangles), Ac (unfilled squares), water after soaking in EtOH (unfilled circles).

Fig. 4. Size distribution of the microcapsules MC160 in various solvents. Symbols indicate the same meanings as Fig. 3.

Fig. 5. Plots of the scattering intensity $I(q)$ vs the absolute value of the scattering vector $q$ for the microcapsules MC110 in EtOH. Solid curves, theoretical values calculated from eqs (1) and (2) with indicated parameters.

Fig. 6. Plots of $I(q)$ vs $q$ for the microcapsules MC160 in EtOH. Solid curves, theoretical values calculated from eqs (1) and (2) with indicated parameters. 
Inner medium (I)

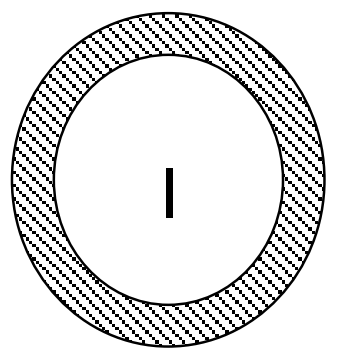

Change the outer medium

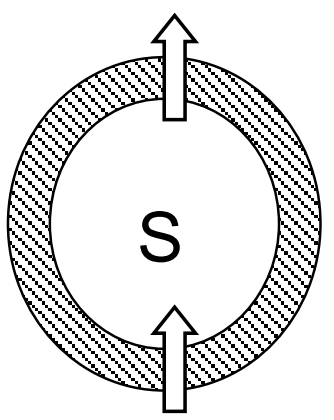

Water

Solvent (S)

Fig. 1. Terao et al. 


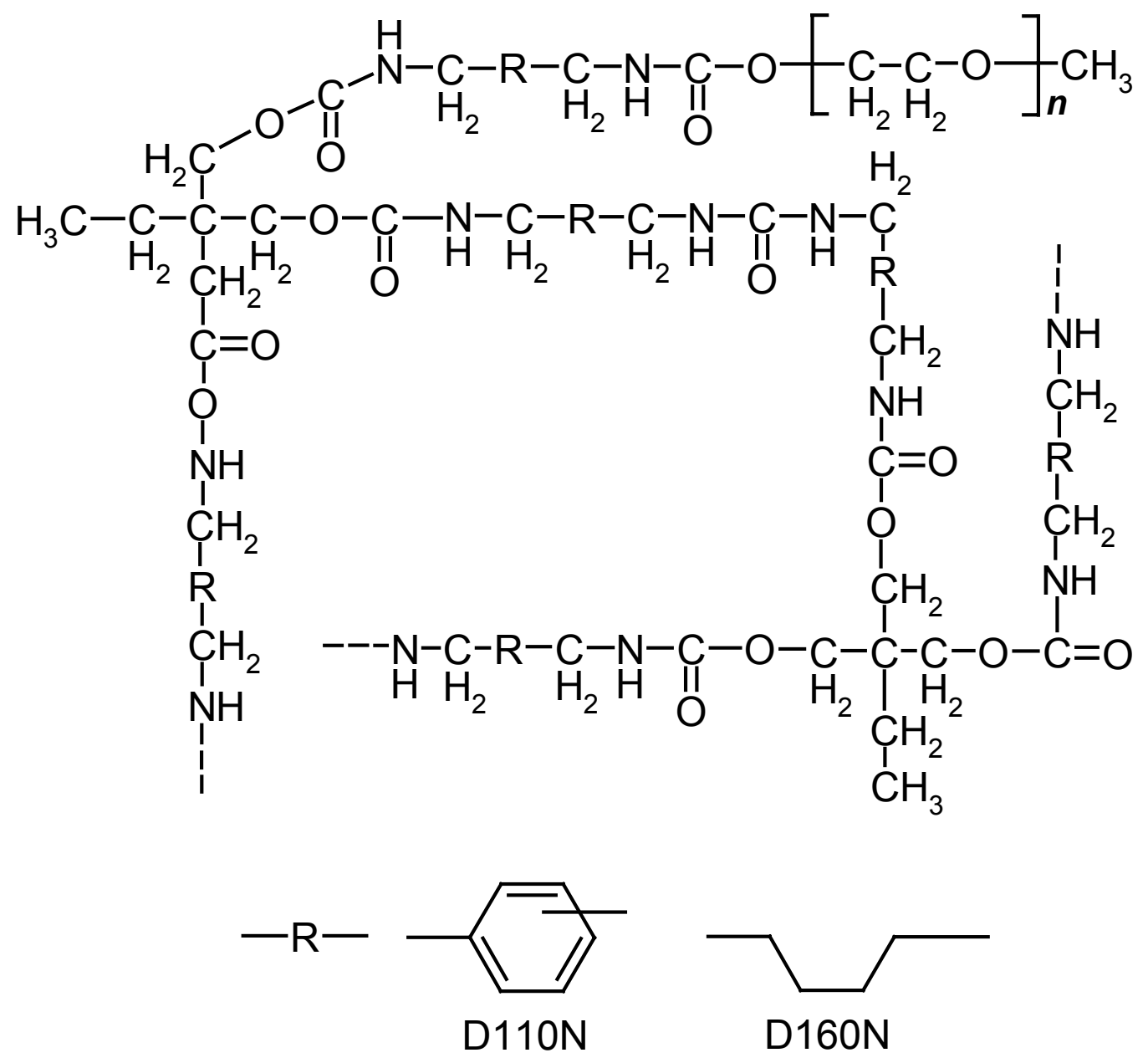

Fig. 2. Terao et al. 


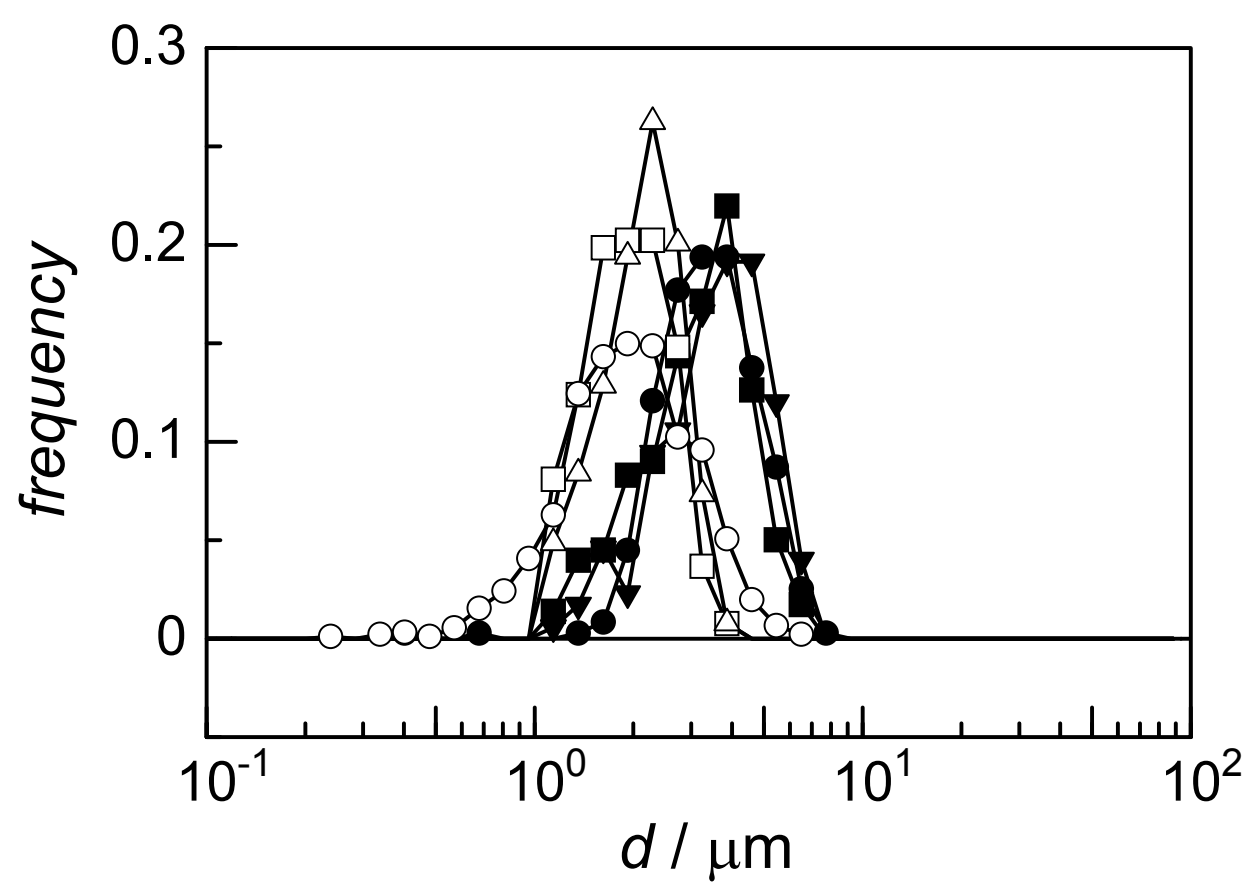

Fig. 3. Terao et al. 


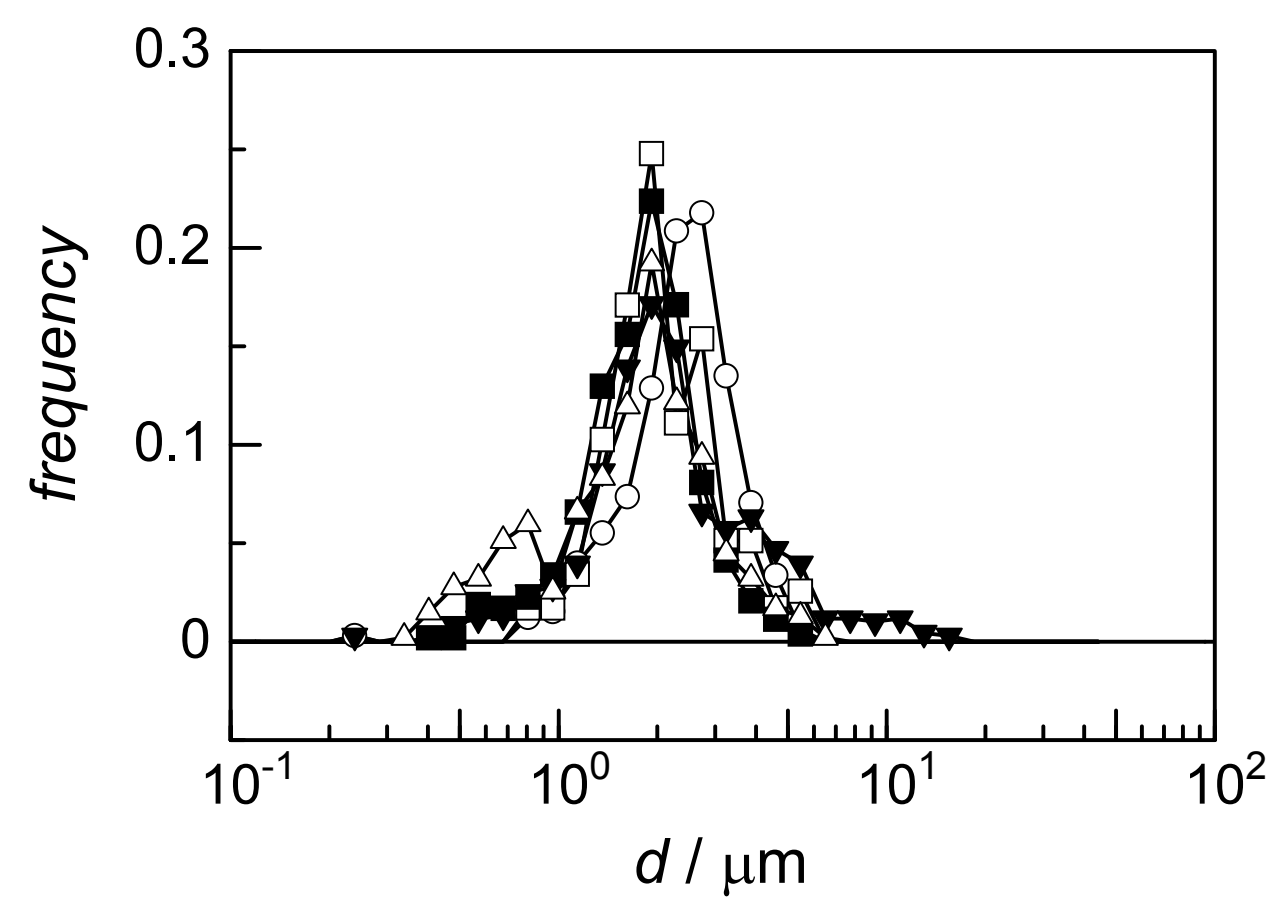

Fig. 4. Terao et al. 


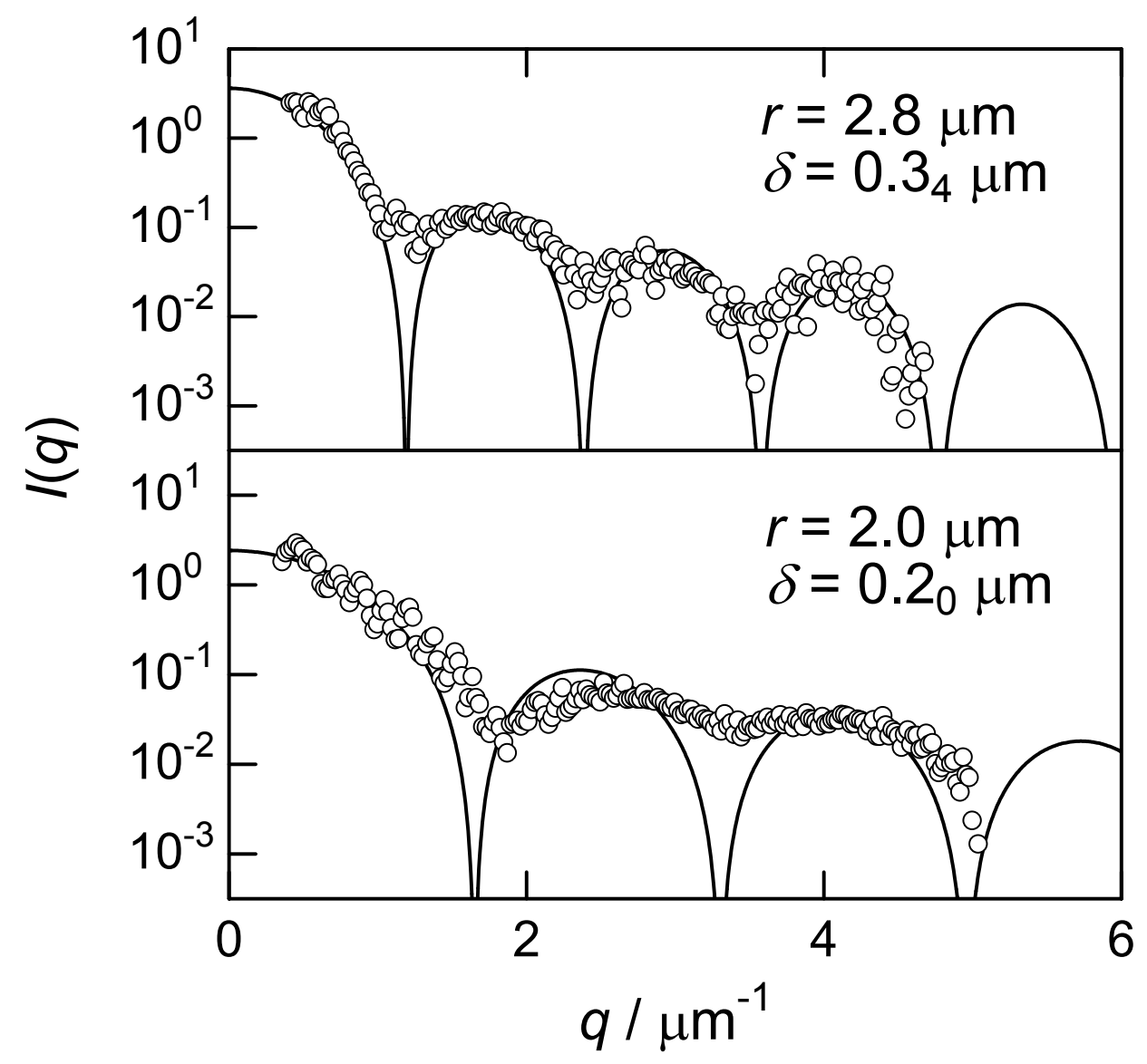

Fig. 5. Terao et al. 


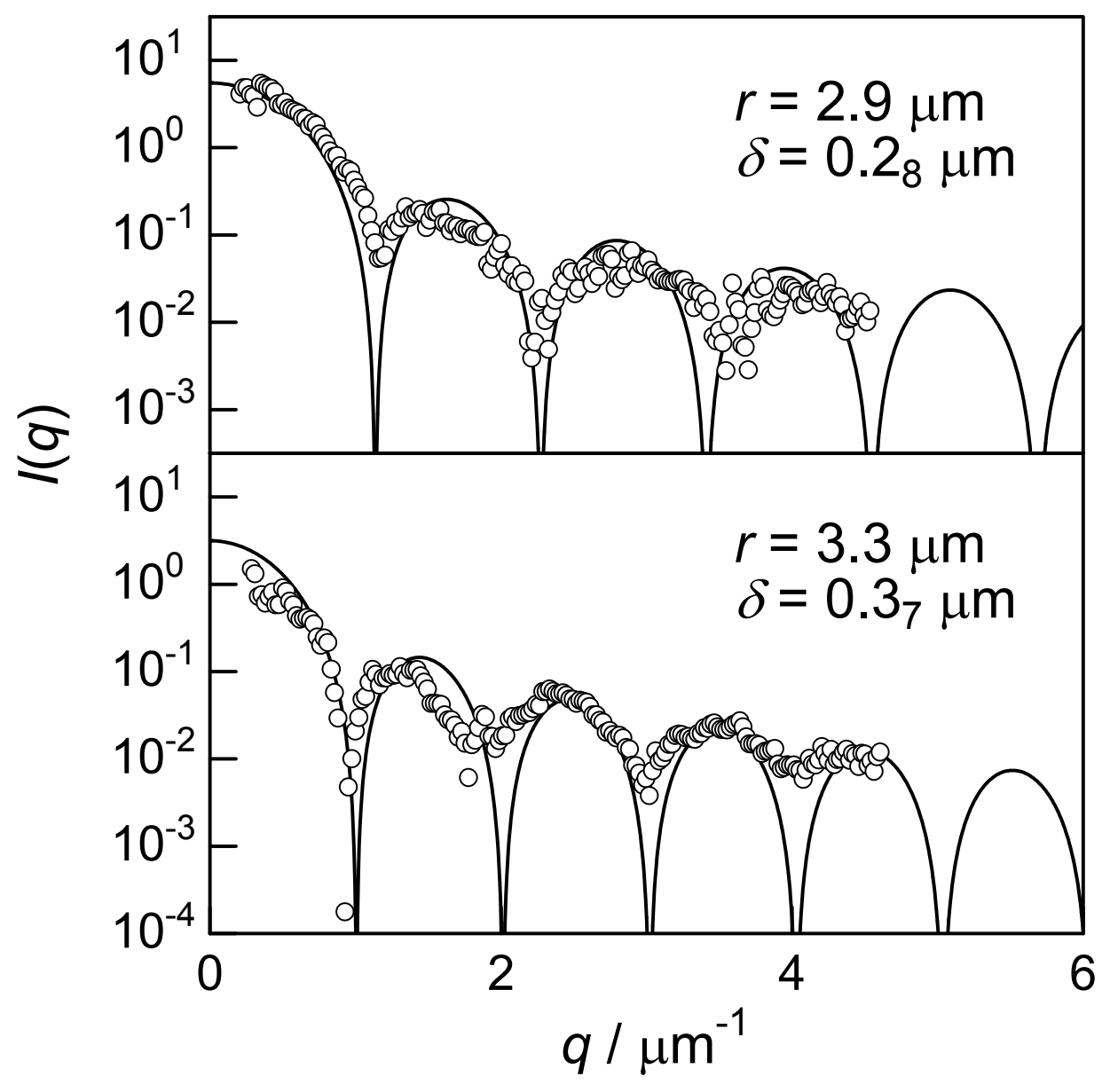

Fig. 6. Terao et al. 